\title{
PREVISÃO DE ANALISTAS E AS ESTRATÉGIAS DE GERENCIAMENTO DE RESULTADOS UTILIZADAS PARA EVITAR SURPRESA NOS LUCROS
}

\section{ANALYST FORECAST AND EARNINGS MANAGEMENT STRATEGIES USED TO AVOID SURPRISE IN PROFITS}

\section{PREVISIÓN DE ANALISTAS Y LAS ESTRATEGIAS DE GESTIÓN DE RESULTADOS UTILIZADAS PARA EVITAR SUPRESIÓN EN LOS BENEFICIOS}

Recebido em: 28/10/2017

Avaliado em: 19/12/2018

Reformulado em:28/02/2018

Aceito para publicação em: 20/05/2019

Publicado em: 14/06/2019

Editor Responsável: Moacir M. Rodrigues Junior

\author{
Jislene Trindade Medeiros ${ }^{1}$ \\ Edilson Paulo ${ }^{2}$ \\ Clayton Levy Lima de Melo ${ }^{3}$ \\ Renato Henrique Gurgel Mota ${ }^{4}$
}

\section{RESUMO}

Este estudo tem por objetivo verificar se as empresas listadas na listadas na B3, no período de 2010 a 2015 gerenciaram seus resultados, simultaneamente, por atividades reais e por accruals discricionários para evitar surpresa nos lucros. A investigação reúne entre 1.617 a 513 observações de empresas não financeiras e seguradoras. Os dados foram coletados no banco de dados da Bloomberg®. A surpresa nos lucros foi identificada por meio do erro de previsão dos analistas financeiros, calculado pela diferença percentual entre o lucro previsto pelos analistas e o lucro real da empresa. As proxies de gerenciamento de resultados contábeis utilizadas foram os níveis anormais de atividades reais e os accruals discricionários, estimados por meio de regressões ano a ano. A partir de regressões pelo método pooling of independente cross section com dummies para ano, os resultados revelaram que os accruals dicionários influenciam positivamente a surpresa nos lucros, sugerindo que quanto maior o erro de previsão dos analistas financeiros, maior o nível de gerenciamento de resultados por escolhas contábeis. Entretanto, o mesmo não se observou em relação ao gerenciamento de resultados por atividades reais. Assim, não foram encontrados indícios de que são adotadas, simultaneamente, o gerenciamento de resultados por atividades reais e por accruals discricionários para evitar surpresa nos lucros. Os resultados fornecem informações a reguladores e investidores ao evidenciar que o atendimento a previsão de lucro dos analistas financeiros não é incentivo para os gestores gerenciarem seus resultados no mercado de capitais brasileiros.

Palavras-chave: Informações contábeis; previsão de analistas; gerenciamento de resultados.

\footnotetext{
${ }^{1}$ Mestre em Ciências Contábeis pela Universidade Federal do Rio Grande do Norte; E-mail: jislenetm@ gmail.com

${ }^{2}$ Doutor em Controladoria e Contabilidade pela Universidade de São Paulo; Professor da Universidade Federal do Rio Grande do Sul; E-mail: e.paulo@uol.com.br

${ }^{3}$ Doutor em Ciências Contábeis pelo Programa Multi-Institucional da Universidade de Brasília, Universidade Federal da Paraíba e com a Universidade Federal do Rio Grande do Norte; Professor da Universidade Federal do Rio Grande do Norte; E-mail: clayton_levy@ hotmail.com

${ }^{4}$ Doutor em Ciências Contábeis pelo Programa Multi-Institucional da Universidade de Brasília, Universidade Federal da Paraíba e com a Universidade Federal do Rio Grande do Norte; Professor da Universidade Federal Rural do Semi-Árido; E-mail: renatohenriq@gmail.com
} 


\section{INTRODUÇÃO}

As práticas de gerenciamento de resultados e seus incentivos têm sido objeto de investigação, em nível nacional e internacional, principalmente identificando indícios de empresas que estão gerenciando resultados (Martinez, 2013). Entretanto, esses estudos costumam considerar e investigar a utilização de uma única estratégia de manipulação da informação contábil para atingir um ou múltiplos incentivos.

De modo divergente, esta pesquisa busca verificar se as empresas com ações negociadas no mercado acionário brasileiro, utilizam simultaneamente mais de uma estratégia de gerenciamento de resultados para alcançar um objetivo específico, partindo do pressuposto de que os gestores podem utilizar, conjuntamente, tanto o gerenciamento de resultados por meio de atividades reais como por accruals discricionários.

Schipper e Vincent (2003) observam que normas de contabilidade mais rígidas podem resultar em uma substituição das estratégias de manipulação dos números contábeis, resultando na redução do gerenciamento de resultados por accruals e em um aumento no gerenciamento de resultados por atividades reais. Paulo (2007), Zang (2011) e Cupertino, Martinez e Costa Jr. (2015), observam que é provável que sejam utilizadas, simultaneamente, mais de uma estratégia de manipulação dos dados contábeis para que seja alcançado o resultado do exercício pretendido.

Para Degeorge, Patel e Zeckauser (1999) uma das principais metas de lucros que os gestores tentam alcançar é a previsão dos analistas financeiros e, conforme Paulo (2007), um dos incentivos relevantes para a manipulação dos números contábeis está associado ao ajustamento dos resultados a essas previsões, visto que, os analistas, além de consolidarem as expectativas do mercado, segundo $\mathrm{Yu}$ (2008), influenciam significativamente o comportamento dos investidores.

Barth, Elliott e Finn (1999); Kasznik e McNichols (2002) e Myers, Myers e Skinner (2007) encontraram evidências de que, as empresas que atingem ou superam as metas de lucros de períodos anteriores ou as expectativas de lucros previstas pelos analistas de forma regular, são recompensadas com maiores valorizações. De modo similar, Brown e Caylor (2005) verificaram que as empresas que conseguem atender ou superar essa meta acabam obtendo maiores retornos, em comparação com firmas semelhantes, que não alcançam os resultados estimados pelos analistas.

Portanto, evitar reportar lucros abaixo das previsões dos analistas é importante para os gestores e também para os participantes do mercado. Dessa forma, tal fato, pode motivar os gestores a utilizarem de modo oportunista sua discricionariedade, gerenciando seus resultados, no que se refere a escolhas contábeis, (Macedo, \& Kelly, 2016) e/ou na manipulação das atividades reais (Reis, Cunha, \& Ribeiro, 2014). Conforme Dechow, Ge e Schrand (2010), é útil investigar se os pequenos erros de previsão estão relacionados com práticas de gerenciamento de resultados.

Assim, diante dos incentivos que as empresas possuem em atingir os resultados previstos pelos analistas financeiros, como forma de satisfazer as expectativas do mercado de capitais e, considerando ser provável que sejam utilizadas mais de uma estratégia de manipulação dos dados contábeis o presente estudo é norteado pelo seguinte questionamento: as empresas de capital aberto listadas na B3 gerenciam seus resultados por atividades reais e por accruals discricionários para evitar surpresa nos lucros?

Dessa forma, o objetivo deste estudo é verificar se as empresas de capital aberto listadas na B3 S.A. - Brasil, Bolsa, Balcão gerenciaram seus resultados por accruals discricionários e por atividades reais para evitar surpresa nos lucros em relação a meta de lucro estabelecida na previsão de consenso dos analistas financeiros. A amostra reúne dados do período de 2010 a 2015. Para a mensuração das proxies das estratégias de gerenciamento de resultados contábeis, foram utilizados: o modelo Paulo (2007), para estimar os accruals discricionários; o modelo Roychowdhury (2006), para estimar os níveis anormais do fluxo de caixa operacional, do volume de produção e das despesas discricionárias; e o modelo McNichols e Wilson (1988), para analisar o relacionamento entre os níveis 
anormais das atividades reais e os níveis dos accruals discricionários das empresas suspeitas de gerenciarem seus resultados com a finalidade de evitar surpresa nos lucros.

Embora no Brasil alguns estudos investiguem a adoção de estratégias de gerenciamento de resultados contábeis e seus incentivos (Cupertino, Martinez, \& Costa, 2015; Reis, Lamounier, \& Bressan, 2015), poucos são os que examinam a utilização simultânea de estratégias de gerenciamento de resultados para atingir o resultado previsto pelos analistas financeiros e atender as expectativas do mercado (Martins, Paulo, \& Monte, 2016; Mota, Silva Filho, Oliveira, \& Paulo, 2017). Os achados também contribuem para a discussão do papel dos analistas financeiros para o mercado de capitais brasileiro e podem interessar aos usuários das demonstrações financeiras, especialmente analistas, auditores e investidores.

\section{REFERENCIAL TEÓRICO}

Quadro 1 - Pesquisas sobre gerenciamento de resultados no Brasil

\begin{tabular}{|c|c|c|}
\hline Referência & Principais Resultados & Método \\
\hline $\begin{array}{l}\text { Martinez e } \\
\text { Cardozo } \\
(2009)\end{array}$ & $\begin{array}{l}\text { O estudo encontrou evidências de que as empresas brasileiras, não } \\
\text { financeiras nem seguradoras, gerenciam os números contábeis mediante } \\
\text { atividades reais (despesas de vendas, gerais e administrativas e pelo nível } \\
\text { de produção) e escolhas contábeis e, que tais escolhas são, em alguns casos, } \\
\text { negativamente correlacionadas. }\end{array}$ & $\begin{array}{l}\text { Adaptado de Anderson, } \\
\text { Banker e Janakiraman } \\
\text { (2003), Roychowdhury } \\
\text { (2006) e KS (1995). }\end{array}$ \\
\hline $\begin{array}{l}\text { Santos, } \\
\text { Verhageme } \\
\text { Bezerra } \\
(2011)\end{array}$ & $\begin{array}{l}\text { A pesquisa apontou evidências de gerenciamento de resultados por meio de } \\
\text { atividades reais tanto aquelas relativas a despesas com vendas, gerais e } \\
\text { administrativas como para os níveis de produção nas indústrias brasileiras } \\
\text { abertas do setor de Siderurgia e Metalurgia. }\end{array}$ & $\begin{array}{l}\text { Anderson, Banker e } \\
\text { Janakiraman (2003) e } \\
\text { Roychowdhury (2006). }\end{array}$ \\
\hline $\begin{array}{l}\text { Ardison, } \\
\text { Martinez e } \\
\text { Galdi (2012) }\end{array}$ & $\begin{array}{l}\text { Os resultados indicaram que não há relação entre o índice de alavancagem } \\
\text { e o gerenciamento de resultados. Além disso, as principais conclusões } \\
\text { sugerem que o aumento da dívida pode reduzir despesas discricionárias e, } \\
\text { por sua vez, reduzir o gerenciamento de resultados por accruals. }\end{array}$ & $\begin{array}{l}\text { Jones (1991), Jones } \\
\text { Modificado (1995) e } \\
\text { KS (1995). }\end{array}$ \\
\hline $\begin{array}{l}\text { Grecco } \\
(2013)\end{array}$ & $\begin{array}{l}\text { Verificou que a adoção das IFRS não restringiu o gerenciamento de } \\
\text { resultadospor accruals discricionários. Os resultados evidenciaram } \\
\text { também, que empresas maiores e mais alavancadas tendem a gerir menos } \\
\text { seus resultados. }\end{array}$ & $\begin{array}{c}\text { Jones Modificado } \\
\text { (1995). }\end{array}$ \\
\hline $\begin{array}{c}\text { Dutra e } \\
\text { Costa }(2014)\end{array}$ & $\begin{array}{l}\text { Foi observado indícios de que o gerenciamento de resultados, por accruals } \\
\text { discricionários, foi utilizado para diminuir a antecipação de resultado e } \\
\text { possivelmente utilizado para melhorar a relação lucro } x \text { retorno, pelas } \\
\text { empresas do Brasil, listadas na bolsa de valores de São Paulo, no período } \\
\text { entre } 1997 \text { a } 2007 \text {. }\end{array}$ & $\begin{array}{c}\text { Jones Modificado } \\
\text { (1995). }\end{array}$ \\
\hline $\begin{array}{l}\text { Barros, } \\
\text { Menezes, } \\
\text { Colauto e } \\
\text { Teodoro } \\
(2014)\end{array}$ & $\begin{array}{l}\text { O coeficiente angular da alavancagem financeira se mostrou negativo em } \\
\text { relação a variável gerenciamento de resultados, indicando que à medida que } \\
\text { aumenta a participação de capital de terceiros por decorrência de } \\
\text { financiamentos, diminui-se a inclinação da gestão em praticar o } \\
\text { gerenciamento de resultados. }\end{array}$ & \\
\hline $\begin{array}{c}\text { Reis, } \\
\text { Lamounier e } \\
\text { Bressan } \\
(2015)\end{array}$ & $\begin{array}{l}\text { As evidências sugerem que no mercado de capitais brasileiro as empresas } \\
\text { evitam divulgar perdas por meio da adoção de gerenciamento das atividades } \\
\text { reais. Contudo, os gestores somente conseguem gerenciar as atividades reais } \\
\text { por meio da redução das despesas operacionais (gerais, vendas e } \\
\text { administrativas). }\end{array}$ & $\begin{array}{c}\text { Roychowdhury (2006), } \\
\text { Gunny (2010) e } \\
\text { Histograma. }\end{array}$ \\
\hline $\begin{array}{l}\text { Cupertino; } \\
\text { Martinez; } \\
\text { Costa Jr. } \\
\text { (2015) }\end{array}$ & $\begin{array}{l}\text { Os resultados apontaram evidências da ocorrência da manipulação de } \\
\text { resultados por decisões operacionais no mercado brasileiro de capitais, que } \\
\text { há uma relação de sequencialidade das estratégias para a manipulação do } \\
\text { resultado do exercício, onde o nível dos accruals depende do montante do } \\
\text { gerenciamento por decisões operacionais. }\end{array}$ & $\begin{array}{c}\text { Roychowdhury (2006), } \\
\text { Gunny (2010), Zang } \\
\text { (2011). }\end{array}$ \\
\hline
\end{tabular}

Fonte: Dados da pesquisa (2017).

Gerenciamento de resultados, de acordo com Healy e Wahlen (1999), é a manipulação das informações contábeis com finalidade oportunista, proveniente de uma ação discricionária dos 
gestores sobre a informação contábil e sobre as atividades reais da empresa para alterar as informações financeiras, de modo a iludir investidores sobre o desempenho econômico da firma.

Zang (2011) evidenciou que os gestores utilizam a manipulação das atividades reais e por escolhas contábeis como estratégias substitutas, alternando entre os dois métodos de gerenciamento de resultados de acordo com os custos relativos a cada estratégia, em que o nível de gerenciamento de resultados por accruals é ajustado de acordo com o nível de gerenciamento das atividades reais da empresa.

No mercado de capitais brasileiro, de modo convergente ao encontrado por Zang (2011), Cupertino, Martinez e Costa Jr. (2015) evidenciaram que as estratégias de gerenciamento de resultados são utilizadas em conjunto e de forma interativa para manipular o resultado do exercício. De acordo com os resultados, há uma relação de substituição entre as estratégias, na qual os administradores gerenciam o resultado por meio de atividades reais durante o exercício físcal e, após o encerramento do exercício, ajustam o nível de accruals discricionários a ser lançado com base no montante realizado de gerenciamento de resultado por meio de atividades reais.

A utilização das duas estratégias como substitutas, pode estar relacionada ao fato levantado por Roychowdhury (2006), de que a manipulação por competência (accruals) possui flexibilidade limitada pelas operações do negócio e pela manipulação dos accruals de períodos anteriores, assim como também, devido aos custos associados a cada estratégia. Portanto, verifica-se que os números contábeis podem ser manipulados simultaneamente visando alcançar ou atender uma motivação particular.

No Brasil, alguns estudos têm apresentado evidências de que as empresas gerenciam seus resultados por meio de atividades reais e por accruals discricionários. Dentre essas pesquisas, cabe mencionar as constantes no Quadro 1.

Quadro 2 - Pesquisas sobre previsão de analistas e gerenciamento de resultados

\begin{tabular}{|c|c|}
\hline Referência & Principais Resultados \\
\hline $\begin{array}{l}\text { Bartov, Givoly } \\
\text { e Hayn (2002) }\end{array}$ & $\begin{array}{l}\text { Evidenciaram que o mercado recompensa com retornos diferenciados as empresas que conseguem } \\
\text { atender ou superar as expectativas dos analistas, habitualmente, visto que, constataram que as } \\
\text { empresas que superam as previsões de lucros dos analistas financeiros obtiveram retornos anormais } \\
\text { no anúncio do resultado e que estes retornos anormais não se reverteram no período seguinte ao do } \\
\text { anúncio. }\end{array}$ \\
\hline $\begin{array}{l}\text { Roychowdhury } \\
\quad(2006)\end{array}$ & $\begin{array}{l}\text { Encontrou evidências de que as empresas manipulam as atividades reais para evitar a divulgação de } \\
\text { perdas e também surpresas negativas em relação as previsões anuais dos analistas, oferecendo } \\
\text { descontos no preço de venda para aumentar, temporariamente, o volume de vendas, reduzindo o } \\
\text { custo do produto vendido (CPV) e os gastos discricionários de modo agressivo para melhorar os } \\
\text { resultados. }\end{array}$ \\
\hline $\begin{array}{l}\text { Martinez } \\
(2006)\end{array}$ & $\begin{array}{l}\text { De acordo com os resultados, o mercado brasileiro reage de modo significante a surpresa no } \\
\text { resultado.No caso de surpresas negativas, o mercado parece antecipar o resultado com retornos } \\
\text { anormais negativos nos períodos que antecedem o anúncio. Já para as surpresas positivas inicia-se } \\
\text { um ciclo de retornos anormais positivos após o anúncio do resultado. }\end{array}$ \\
\hline $\begin{array}{l}\text { Martinez } \\
\text { (2007) }\end{array}$ & $\begin{array}{l}\text { Evidenciou que os analistas de mercado que realizam previsões de empresas brasileiras têm } \\
\text { comportamentos enviesados e suas projeções, em média, são otimistas. }\end{array}$ \\
\hline $\begin{array}{l}\text { Ayers, Jiang e } \\
\text { Yeung (2006) }\end{array}$ & $\begin{array}{l}\text { Verificaram um relacionamento significante e positivo entre os accruals discricionários e } \\
\text { atendimento ao lucro previsto pelos analistas financeiros e que quanto menor o erro de previsão mais } \\
\text { essa relação se intensifica. }\end{array}$ \\
\hline $\begin{array}{l}\text { Martinez } \\
(2011)\end{array}$ & $\begin{array}{l}\text { Os resultados indicaram existir uma correlação negativa entre o número de analistas e a magnitude } \\
\text { dos accruals discricionários em termos absolutos, indicando que a cobertura de analista inibe o } \\
\text { gerenciamento de resultados. }\end{array}$ \\
\hline $\begin{array}{l}\text { Martins, Paulo } \\
\text { e Monte } \\
\quad(2016)\end{array}$ & $\begin{array}{l}\text { De acordo com os resultados, o volume médio dos accruals discricionários relaciona-se } \\
\text { positivamente com o erro médio da previsão. Contudo, quando os resultados da empresa estão } \\
\text { distantes da previsão o volume de accruals é menos elevado, indicando menor nível de } \\
\text { gerenciamento de resultados contábeis, sugerindo que os gestores são mais incentivados a gerenciar } \\
\text { resultados quando estão próximos de atingir a previsão dos analistas. }\end{array}$ \\
\hline
\end{tabular}

Fonte: Dados da pesquisa (2017). 
Alguns estudos fornecem evidências de que os gestores gerenciam seus resultados com a finalidade de evitar reportar surpresa para o mercado sobre os lucros e, de acordo com Bartov, Givoly e Hayne (2002) dentre as motivações para que esse benchmark seja alcançado incluem: maximizar o preço das ações, aumentar a credibilidade da administração, e evitar custos com litígios que poderiam ser desencadeados por surpresas desfavoráveis nos lucros.

No Quadro 2 é apresentado alguns dos principais estudos realizados sobre o assunto.

Como pode ser observado, para o contexto brasileiro, há evidências de que a cobertura de analistas inibe a prática de gerenciamento de resultados por escolhas contábeis (Martinez, 2011), e indícios de que as empresas gerenciam seus lucros quando estão próximas de alcançar o consenso da previsão dos analistas (Martins, Paulo, \& Monte, 2016). Entretanto, ainda não foi investigado se os gestores gerenciam, conjuntamente, seus resultados por atividades reais e por accruals discricionários para atingir as previsões de lucros dos analistas financeiros.

Assim, formula-se a seguinte hipótese de pesquisa:

$H_{1}$ : as empresas gerenciam seus resultados por atividades reais e por accruals discricionários para evitar surpresa nos lucros.

\section{PROCEDIMENTOS METODOLÓGIOS}

\subsection{Amostra e coleta de dados}

A amostra desse estudo é composta pelas empresas listadas na B3 S.A. - Brasil, Bolsa, Balcão. A escolha do mercado de ações brasileiro se justifica pelo fato deste mercado possuir cobertura de analistas financeiros, o que viabiliza a execução do estudo e, pelo motivo de que os estudos nacionais ainda não investigaram de modo satisfatório a importância desses intermediários de informações para este mercado de capitais, principalmente, ao ponto de motivar os gestores a gerenciarem seus resultados para atender as previsões de lucros por eles proferidas.

Os dados foram coletados nos bancos de dados da Bloomberg® em bases anuais. O período de tempo utilizado para análise foi de 2010 a 2015, totalizando 6 anos. A definição deste período de estudo foi devido à adoção obrigatória e integral das IFRS (International Financial Reporting Standards) pelas empresas brasileiras de capital aberto ter iniciado em 2010, favorecendo, assim, a comparabilidade dos dados.

Ressalte-se que algumas regressões possuem diferentes números de observações, haja vista que algumas empresas não possuem todas as variáveis em todos os anos para todos os modelos utilizados. Assim, cada um dos modelos reúne entre 1.617 a 513 observações.

\subsection{Modelos de gerenciamento de resultados contábeis}

Para se atingir o objetivo desta pesquisa, faz-se necessário estimar o gerenciamento de resultados por atividades reais (GRAR) e por accruals discricionários (GRAD). Os modelos utilizados como proxies para essas duas estratégias de gerenciamento de resultados são apresentados a seguir.

\subsubsection{Modelo de detecção de gerenciamento de resultados por atividades reais}

Para estimação do total do gerenciamento de resultados por atividades reais (GRAR), inicialmente foi utilizado o Modelo Roychowdhury (2006) que estima os padrões anormais dos Fluxos de Caixa Operacionais - $A F C O$, das Despesas Discricionárias - ADD e dos Custos de Produção - APROD, com regressões pelo método pooling of independente cross sections ano a ano, obtido pela diferença do valor real observado e a estimativa obtida por meio das seguintes Equações:

$$
\begin{array}{cr}
\mathrm{FCO}_{\mathrm{it}} / \mathrm{A}_{\mathrm{it}-1}=\alpha_{0+} \alpha_{1}\left(1 / \mathrm{A}_{\mathrm{it}-1}\right)+\beta_{1}\left(\mathrm{~V}_{\mathrm{it}} / \mathrm{A}_{\mathrm{it}-1}\right)+\beta_{2}\left(\Delta \mathrm{V}_{\mathrm{it}} / \mathrm{A}_{\mathrm{it}-1}\right)+\varepsilon_{\mathrm{it}} & 1 \\
\mathrm{PROD} & \mathrm{it} / \mathrm{A}_{\mathrm{it}-1}=\alpha_{0+} \alpha_{1}\left(1 / \mathrm{A}_{\mathrm{it}-1}\right)+\beta_{1}\left(\mathrm{~V}_{\mathrm{it}} / \mathrm{A}_{\mathrm{it}-1}\right)+\beta_{2}\left(\Delta \mathrm{V}_{\mathrm{it}} / \mathrm{A}_{\mathrm{it}-1}\right)+\beta_{3}\left(\Delta \mathrm{V}_{\mathrm{it}-1} / \mathrm{A}_{\mathrm{it}-1}\right)+\varepsilon_{\mathrm{it}}
\end{array}
$$


Em que:

$$
\mathrm{DD}_{\mathrm{it}} / \mathrm{A}_{\mathrm{it}-1}=\alpha_{0}+\alpha_{1}\left(1 / \mathrm{A}_{\mathrm{it}-1}\right)+\beta\left(\mathrm{V}_{\mathrm{it}-1} / \mathrm{A}_{\mathrm{it}-1}\right)+\varepsilon_{\mathrm{it}}
$$

$F C O_{i t}=$ fluxo de Caixa Operacional da empresa $i$ no período $t$ (lucro operacional - accruals);

$A_{i t}=$ total do Ativo da empresa $i$ no período $t$;

$P R O D_{i t}=$ custo de produção da empresa $i$ no período $t$ (custo do produto vendido $+\Delta$ estoque);

$\mathrm{DD}=$ Despesas discricionárias;

$V_{i t}=$ receita operacional líquida da empresa $i$ no período $t$;

$\Delta V_{i t}=$ variação em vendas da empresa $i$ no período $t$;

$\varepsilon_{i t}=$ é o termo de erro da regressão.

Baseado nos valores estimados, estimou-se o GRAR como proposto por Gunny (2010) que construiu uma medida agregada das três proxies de gerenciamento de resultados por atividades reais, conforme a seguinte Equação:

$$
\mathrm{GRAR}_{\mathrm{it}}=\left(\mathrm{AFCO}_{\mathrm{it}}+\mathrm{ADD}_{\mathrm{it}}\right)+\left(\left(\mathrm{APROD}_{\mathrm{it}} \mathrm{X}(-1)\right)\right.
$$

Segundo Gunny (2010), o nível anormal de produção - APROD é multiplicado por -1 para manter o mesmo efeito dos níveis anormais de fluxo de caixa $-A F C O$ e das despesas com vendas, gerais e administrativas $-A D D$.

\subsubsection{Modelo de detecção de gerenciamento de resultados por accruals discricionários}

Para detectar o gerenciamento de resultados por accruals discricionários, o primeiro passo foi estimar accruals totais apurados sob a abordagem do Balanço Patrimonial. Em seguida foram estimados os parâmetros da regressão pelo método pooling of independente cross sections ano a ano, referente aos accruals totais, conforme o modelo proposto por Paulo (2007), segundo a seguinte Equação:

$$
\begin{gathered}
\mathrm{TA}_{\mathrm{i}, \mathrm{t}}=\alpha+\beta_{1} \mathrm{R}_{\mathrm{it}}+\beta_{2} \mathrm{AI}_{\mathrm{it}}+\lambda_{1} \mathrm{FCO}_{\mathrm{it}}+\lambda_{2} \mathrm{E}_{\mathrm{it}}+\lambda_{3} \mathrm{E}_{\mathrm{it}}^{2}+\lambda_{4} \Delta \mathrm{E}_{\mathrm{it}-1}+\lambda_{5} \mathrm{D} \Delta \mathrm{E}_{\mathrm{it}-1}+\lambda_{6} \Delta \mathrm{E}_{\mathrm{it}-1} * \\
\mathrm{D} \Delta \mathrm{E}_{\mathrm{it}-1}+\lambda_{7} \mathrm{TA}_{\mathrm{it}-1}+\mathrm{y}_{1} \mathrm{ab}_{-} \operatorname{Prod}_{\mathrm{it}}+\mathrm{y}_{2} \mathrm{ab}_{-} \mathrm{DD}_{\mathrm{it}}+\varepsilon_{\mathrm{it}}
\end{gathered}
$$

Em que:

$T A_{i t}=$ accruals totais da empresa $i$ no período $t$

$R_{i t}=$ receita das vendas líquidas da empresa $i$ no período $t$, ponderadas pelos ativos totais no final do período $t-1$;

$A I_{i t}=$ ativo imobilizado da empresa $i$ no final do período $t$, ponderado pelos ativos totais no final do período $t-1$;

$F C O_{i t}=$ fluxo de caixa operacional da empresa $i$ no período $t$, ponderado pelos ativos totais no final do período $t-1$;

$E_{i t} \quad=$ resultado contábil da empresa $i$ no período $t$, ponderadas pelos ativos totais no final do período $t-1$;

$\Delta E_{i t-1}=$ variação no lucro líquido contábil da empresa $i$ no ano $t-2$ para o ano $t-1$, ponderadas pelos ativos totais no início do ano $t-2$;

$D \Delta E_{i t-1}=$ variável dummy para indicar se existe variação negativa no lucro líquido contábil da empresa $i$ no ano $t$-2 para o ano $t$-lassumindo valor 1 se $\Delta N I_{i t}<0$, e 0 nos demais casos;

$T A_{t-1}=$ accruals totais da empresa $i$ no período $t$-1, ponderados pelos ativos totais no final do período $t-2$;

$a b \_$Prod $_{i t}=$ comportamento anormal dos custos de produção da empresa $i$ no período $t$;

$a b \_D D_{i t}=$ comportamento anormal das despesas operacionais da empresa $i$ no período $t$;

$\varepsilon_{i t}=$ é o termo de erro da regressão. 
Após a estimação dos parâmetros da regressão e dos accruals totais, os accruals foram decompostos em discricionários (gerenciados) e não discricionários (não gerenciados), calculados pela diferença entre os accruals totais e os accruals não-discricionários, de acordo com a equação proposta por McNichols e Wilson (1988):

$$
\mathrm{AD}_{\mathrm{it}}=\mathrm{TA}_{\mathrm{it}}-\mathrm{NDA}_{\mathrm{it}}
$$

\subsection{Incentivo ao gerenciamento de resultados contábeis}

Para verificar se o comportamento das proxies de gerenciamento contábil - nível anormal do fluxo de caixa (AFCO), nível anormal de despesas discricionárias (ADD), nível anormal dos custos de produção (APROD) e nível dos accruals discricionários está relacionado ao erro de previsão dos analistas financeiros, a análise foi realizada com base no modelo proposto por McNichols e Wilson (1988), conforme descrito abaixo:

$$
\mathrm{GR}_{\mathrm{it}}=\alpha+\beta_{l}(\mathrm{PART})_{\mathrm{it}}+\sum \gamma k X k t+\varepsilon_{\mathrm{it}}
$$

Em que:

$G R_{i t}=$ nível anormal de atividades reais (GRAR) e nível dos accruals discricionários (GRAD) da empresa i no período t;

$P A R T_{i t}=$ variável dummy que representa o incentivo que leva os gestores a se envolverem com práticas de gerenciamento de resultados contábeis, que, nesta pesquisa, assume o valor 1 para as empresas com erro de previsão de até $5 \%$, e valor 0 para as demais empresas;

$X k t=$ outros fatores que influenciam o comportamento dos níveis anormais de atividades reais e dos accruals discricionários da empresa $i$ no período $t$;

$\varepsilon_{i t}=$ é o termo de erro da regressão.

Conforme McNichols e Wilson (1988) se o $\beta$ estimado é significativamente maior que zero, pode-se dizer que existem evidências de que as empresas gerenciam os resultados contábeis por atividades reais e por accruals discricionários relacionadas a hipótese investigada.

No modelo geral, Xkt corresponde a outros fatores que influenciam o gerenciamento de resultados, aqui representado pelas variáveis de controle descritas no Quadro 3, quais sejam, tamanho, ROA, alavancagem, tangibilidade, Market-to-book e big4.

\subsection{Teste da hipótese}

Esta pesquisa buscou investigar o relacionamento entre o atendimento as previsões dos analistas financeiros no mercado de capitais brasileiro e o gerenciamento de resultados contábeis, partindo do pressuposto de que para atingir essa meta, o resultado contábil reportado pelas empresas pode ser, conjuntamente, gerenciado por accruals discricionários e por atividades reais. Desse modo, o modelo a ser utilizado neste estudo pode ser expresso pela seguinte Equação:

$$
\begin{gathered}
\mid \text { Surpresa }_{i t} \mid=\beta_{0}+\beta_{1} G R A R_{i t}+\beta_{2} G R A D_{i t}+\beta_{3} T A M_{i t}+\beta_{4} A L V_{i t}+\beta_{5} R O A_{i t}+\beta_{6} T A N G_{i t}+ \\
\beta_{7 M T B_{i t}}+\beta_{8} B I G 4_{i t}+\sum Y_{n} A n o_{i t}+\varepsilon_{i t}
\end{gathered}
$$

Optou-se pela utilização das variáveis de controle apresentadas no Quadro 3, visto que, segundo Watts e Zimmerman (1990) existe relação entre as escolhas contábeis e as características econômicas de uma empresa e devido estas já terem sido utilizadas em pesquisas anteriores sobre gerenciamento de resultados de alguns autores, dentre eles Cupertino; Martinez; Costa Jr. (2015), Gunny (2010), Paulo (2007), Paulo e Mota (2018), Roychowdhury (2006), Reis, Lamounier e Bressan (2015) e Zang (2011). 
Quadro 3 - Descrição das variáveis, operacionalização e base teórica

\begin{tabular}{|c|c|c|c|}
\hline Nome & Sigla & Operacionalização & Base teórica \\
\hline Surpresa & Surpresa & 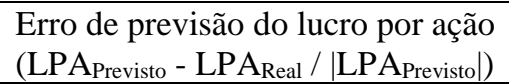 & $\begin{array}{l}\text { Martins, Paulo e Monte (2016), } \\
\text { Mota et al. (2017) }\end{array}$ \\
\hline $\begin{array}{l}\text { Gerenciamento de } \\
\text { resultados por atividades } \\
\text { reais }\end{array}$ & GRAR & $\begin{array}{l}\text { Estimado pelos modelos Gunny } \\
\text { (2010) e Roychowdhury (2006) }\end{array}$ & $\begin{array}{l}\text { Cupertino; Martinez; Costa Jr. } \\
\text { (2015), Reis, Lamounier e Bressan } \\
\text { (2015), Zang (2011) }\end{array}$ \\
\hline $\begin{array}{l}\text { Gerenciamento de } \\
\text { resultados por accruals } \\
\text { discricionários }\end{array}$ & GRAD & $\begin{array}{l}\text { Estimados pelos modelos } \\
\text { McNichols e Wilson (1988) e } \\
\text { Paulo (2007) }\end{array}$ & $\begin{array}{l}\text { Mota et al. (2017), Paulo e Mota } \\
(2018)\end{array}$ \\
\hline \multicolumn{4}{|c|}{ Variáveis de Controle } \\
\hline Tamanho & TAM & Logaritmo natural do ativo total. & $\begin{array}{l}\text { Gunny (2010), Paulo e Mota } \\
\text { (2018) e Roychowdhury (2006) }\end{array}$ \\
\hline Alavancagem & ALV & $\begin{array}{l}\text { Dívida total dividida pelo ativo } \\
\text { total }\end{array}$ & $\begin{array}{l}\text { Almadi e Lazic (2016) } \\
\text { Call et al. (2014) }\end{array}$ \\
\hline Rentabilidade & ROA & $\begin{array}{l}\text { Lucro líquido dividido pelo ativo } \\
\text { total }\end{array}$ & $\begin{array}{l}\text { Bekiris e Doukakis (2011), Bowen, } \\
\text { Rajgopal e Venkatachalam (2008) }\end{array}$ \\
\hline Tangibilidade & TANG & $\begin{array}{l}\text { Ativo imobilizado dividido pelo } \\
\text { ativo total }\end{array}$ & Persakis e Iatridis (2015) \\
\hline Market to book & MTB & $\begin{array}{l}\text { Valor de mercado das ações } \\
\text { dividido pelo PL. }\end{array}$ & $\begin{array}{l}\text { Gunny (2010), Lamounier e } \\
\text { Bressan (2015) e Roychowdhury } \\
(2006)\end{array}$ \\
\hline Qualidade da auditoria & BIG4 & $\begin{array}{l}\text { Dummy que assume } 1 \text { para as } \\
\text { empresas auditadas por uma das } 4 \\
\text { maiores empresas de auditoria do } \\
\text { mundo e } 0 \text { nos demais casos }\end{array}$ & Becker et al. (1998), Koh (2003) \\
\hline Ano & ANO & Dummy indicando o ano. & Koh (2003) \\
\hline
\end{tabular}

Fonte: Dados da pesquisa (2017).

\section{APRESENTAÇÃO E ANÁLISE DOS RESULTADOS}

\subsection{Análise do gerenciamento de resultados por atividades reais}

Para estimar o gerenciamento de resultados total por atividades reais (GRAR), primeiramente, foram estimados os coeficientes dos comportamentos normais das despesas discricionárias (DD), dos fluxos de caixa (FCO) e dos custos de produção (PROD). Na Tabela 1 são apresentados os coeficientes médios entre os anos e as estatísticas $t$ dos erros padrão da média em todos os anos, assim como as médias dos $\mathrm{R}^{2}$.

Tabela 1 - Estatística descritiva e estimação dos níveis normais de atividades reais

\begin{tabular}{|c|c|c|c|c|c|c|}
\hline \multirow{2}{*}{ Variáveis } & \multicolumn{2}{|c|}{ Estatística descritiva } & \multirow{2}{*}{$\begin{array}{c}\text { Sinal } \\
\text { Esperado }\end{array}$} & \multirow{2}{*}{$\begin{array}{l}\text { FCO } \\
\text { Coef. }\end{array}$} & \multirow{2}{*}{$\begin{array}{c}\text { PROD } \\
\text { Coef. }\end{array}$} & \multirow{2}{*}{$\begin{array}{c}\text { DD } \\
\text { Coef. }\end{array}$} \\
\hline & Média & Desvio-padrão & & & & \\
\hline $1 / \mathbf{A}_{\mathrm{it}-1}$ & 0,013 & 0,133 & $?$ & $-0,949 * * *$ & 0,040 & $0.451 * * *$ \\
\hline $\mathbf{V}_{\text {it }} / \mathbf{A}_{\text {it-1 }}$ & 0,751 & 0,644 & + & 0,020 & $0,831 * * *$ & - \\
\hline $\mathbf{V}_{\text {it-1 }} / \mathbf{A}_{\text {it-1 }}$ & 0,677 & 0,571 & + & - & - & $0.139 * * *$ \\
\hline$\Delta \mathbf{V}_{\text {it }} / \mathbf{A}_{\text {it-1 }}$ & 0,040 & 0,289 & + & $-0,013$ & $-0,104 *$ & - \\
\hline$\Delta \mathbf{V}_{\text {it - } 1} / \mathbf{A}_{\mathrm{it}-1}$ & 0,413 & 0,356 & + & - & $-0,020 *$ & - \\
\hline Constante & - & - & $?$ & $0,060 * * *$ & $-0,078 * * *$ & $0.060 * * *$ \\
\hline \multicolumn{7}{|c|}{ Descrição } \\
\hline \multicolumn{4}{|c|}{ Observações } & 1.590 & 1.500 & 1.590 \\
\hline \multicolumn{4}{|c|}{$\mathbf{F}(p-v a l o r)$} & $78,628 * * *$ & $744,380 * * *$ & $38,040 * * *$ \\
\hline \multicolumn{4}{|c|}{$\mathbf{R}^{2}$} & 0,350 & 0,919 & 0,218 \\
\hline \multicolumn{4}{|c|}{$\mathbf{R}^{2}$ Ajustado } & 0,343 & 0,918 & 0,212 \\
\hline
\end{tabular}

*,** e *** denotam, respectivamente, significância de $10 \%, 5 \%$ e $1 \%$.

Regressões pelo método pooling of independente cross section, estimadas ano a ano.

Fonte: Resultados da pesquisa (2017). 
Observa-se na Tabela 1, que nos resultados do modelo de estimação do comportamento normal dos fluxos de caixa (FCO), apenas a variável " $1 / \mathrm{A}_{\mathrm{it}-1}$ " apresentou significância estatística a $1 \%$. Observa-se que a variável receita líquida apresentou coeficiente positivo e não significante diferentemente do encontrado por Roychowdhury (2006), Cupertino; Martinez; Costa Jr. (2015) e Reis, Lamounier e Bressan (2015).

Com relação à variável variação da receita líquida, também foram estimados coeficientes não significantes, o que corrobora o verificado por Cupertino; Martinez; Costa Jr. (2015) e por Reis, Lamounier e Bressan (2015). Contudo, contrário ao resultado de Roychowdhury (2006), que encontrou resultado positivo e significativo a $10 \%$. Dessa forma, uma variação nessas variáveis, não implica em variação positiva no Fluxo de Caixa Operacional do período corrente, resultado contrário ao esperado, na presença de gerenciamento de resultados.

Em relação as variáveis do nível de produção, verifica-se que o coeficiente da variável Receita Líquida $\left(\mathrm{V}_{\mathrm{it}} / \mathrm{A}_{\mathrm{it}-1}\right)$ mostrou-se positivo em 0,831 e significante a $1 \%$, o que significa que um aumento na receita líquida em uma unidade, provocará um aumento de 0,831 no nível de produção. Esses resultados são similares ao encontrado por Roychowdhury (2006), Martinez e Cardoso (2009), Gunny (2010), Verhagem, Santos e Bezerra (2011), Zang (2011), Cupertino; Martinez; Costa Jr. (2015) e Reis, Lamounier e Bressan (2015).

Observa-se que o valor do coeficiente obtido para a variação da Receita Líquida $\left(\Delta \mathrm{V}_{\mathrm{it}} / \mathrm{A}_{\mathrm{it}-1}\right)$ e variação da receita líquida do período anterior $\left(\Delta \mathrm{V}_{\mathrm{it}-1} / \mathrm{A}_{\mathrm{it}-1}\right)$, foram 0,104 e 0,020 negativos, respectivamente, e também significativos ao nível de $10 \%$. Desse modo, quando o volume das vendas aumenta, os níveis de produção diminuem, o que tecnicamente não deveria ocorrer, visto que com o aumento das vendas os níveis de produção também deveriam aumentar, indicando que as empresas utilizam os níveis de produção para aumentar os resultados contábeis.

Esse resultado está alinhado ao verificado por Verhagem, Santos e Bezerra (2011) e Reis, Lamounier e Bressan (2015). Todavia, tais resultados são divergentes aos observados nos trabalhos de Roychowdhury (2006), Martinez e Cardoso (2009), Gunny (2010), Zang (2011) e Cupertino; Martinez; Costa Jr. (2015) em que foi encontrado um sinal positivo para os coeficientes destas variáveis.

No que tange aos níveis normais de despesas discricionárias, verifica-se na Tabela 1 , que o coeficiente da variável Receita Líquida se mostrou positivo em 0,139 e significante ao nível de 1\%, resultado esse similar ao encontrado por Roychowdhury (2006), Zang (2011), Cupertino, Martinez e Costa Jr. (2015) e Reis, Lamounier e Bressan (2015). Conforme esses resultados, um aumento na receita líquida em uma unidade, resultará em um aumento em 0,139 nas despesas discricionárias.

Esses resultados fornecem evidências contrárias ao gerenciamento de resultados das Despesas Discricionárias para aumentar os resultados contábeis, uma vez que as despesas discricionárias estão acompanhando o volume de vendas das empresas.

Dessa forma, foram encontrados indícios de que os níveis de produção (PROD) são utilizados com a finalidade de gerenciar os resultados. Porém, no que concerne ao Fluxo de Caixa Operacional (FCO) e as Despesas Discricionárias (DD) os resultados fornecem evidências contrárias ao gerenciamento de resultados.

\subsection{Análise do gerenciamento de resultados por accruals discricionários}

Para detecção do gerenciamento de resultados por accruals discricionários (GRAD), foi utilizado o modelo Paulo (2017). Este modelo considera que o nível dos accruals discricionários são afetados pela manipulação das atividades reais. Desse modo, são utilizadas variáveis relacionadas aos comportamentos anormais das atividades reais, já calculadas anteriormente pelo modelo Roychowdhury (2006). Na Tabela 2 são apresentados os coeficientes médios das regressões estimadas a cada ano e a estatística $t$ calculada usando o erro padrão da média em todos os anos, como também os $\mathrm{R}^{2}$ médios. 
PREVISÃO DE ANALISTAS E AS ESTRATÉGIAS DE GERENCIAMENTO DE RESULTADOS UTILIZADAS PARA EVITAR SURPRESA NOS LUCROS

Tabela 2 - Estatística descritiva e estimação dos níveis normais dos accruals totais

\begin{tabular}{|c|c|c|c|c|}
\hline \multirow{2}{*}{ Variáveis } & \multicolumn{2}{|c|}{ Estatística descritiva } & \multicolumn{2}{|c|}{ Regressão } \\
\hline & Média & Desvio-padrão & Sinal Esperado & Coeficientes \\
\hline $\mathbf{R}_{\text {it }}$ & 0,751 & 0,644 & + & 0,005 \\
\hline Imobit $_{\text {it }}$ & 0,315 & 0,309 & - & $-0,033$ \\
\hline FCO $_{\text {it }}$ & 0,550 & 0,297 & - & $-0,581 * * *$ \\
\hline $\mathbf{E}_{\text {it }}$ & $-0,018$ & 0,368 & + & $0,665 * * *$ \\
\hline $\mathbf{E}^{2}$ it & 0,119 & 1,74 & $?$ & 0,055 \\
\hline$\Delta \mathbf{E}_{\mathrm{it}-1}$ & 0,002 & 0,248 & $?$ & 0,028 \\
\hline $\mathbf{D} \Delta \mathbf{E}_{\text {it }}$ & - & - & $?$ & 0,015 \\
\hline$\Delta \mathbf{E}_{\mathrm{it}-1} * \mathbf{D} \Delta \mathbf{E}_{\mathrm{it}-\mathbf{1}}$ & $-0,046$ & 0,177 & - & 0,204 \\
\hline $\mathbf{T} \mathbf{A}_{\mathrm{it}-\mathbf{1}}$ & $-0,018$ & 0,249 & - & $-0,092$ \\
\hline ab_Prodit $_{\text {it }}$ & 0,000 & 0,159 & + & 0,140 \\
\hline ab_dd it $_{\text {it }}$ & 0,000 & 0,199 & + & 0,326 \\
\hline Constante & - & - & $?$ & 0,006 \\
\hline \multicolumn{5}{|c|}{ Descrição } \\
\hline \multicolumn{4}{|c|}{ Observações } & 1.344 \\
\hline \multicolumn{4}{|c|}{ F (p-valor $)$} & $16,147 * * *$ \\
\hline \multicolumn{4}{|c|}{$\mathbf{R}^{2}$} & 0,440 \\
\hline \multicolumn{4}{|c|}{$\mathbf{R}^{2}$ Ajustado } & 0,411 \\
\hline
\end{tabular}

*,** e $* * *$ denotam, respectivamente, significância de $10 \%, 5 \%$ e $1 \%$.

Regressões pelo método pooling of independente cross section, estimadas ano a ano.

Fonte: Resultados da Pesquisa (2017).

Como pode ser verificado na Tabela 2, o modelo Paulo apresentou um $\mathrm{R}^{2}$ médio de $44 \%$. Verifica-se que as variáveis " $F C O_{i t}$ " e " $E_{i t}$ " apresentaram coeficiente estatisticamente significantes a $1 \%$ e, os sinais encontrados para os coeficientes dessas variáveis foram os esperados. Dessa forma, evidencia-se que quando há uma relação inversa entre o Fluxo de caixa Operacional corrente e os accruals totais, assim como existe uma relação direta entre os accruals totais e o resultado contábil corrente.

Diferente do encontrado por Paulo (2007), no contexto brasileiro, o sinal da variável correspondente aos accruals totais defasados também foram os esperados, indicando que os accruals do período anterior são revertidos no período corrente, contudo o coeficiente dessa variável não foi estatisticamente significante. Em relação ao comportamento anormal das despesas discricionárias (ad_DD) e dos níveis de produção (ab_Prod), os sinais encontrados foram os esperados, indicando que um aumento nessas variáveis leva a um aumento nos accruals totais e, como essas variáveis não apresentaram significância estatística, parte dos accruals discricionários totais encontrados não são decorrentes do gerenciamento das atividades reais dos níveis de produção e das despesas discricionárias.

\subsection{Níveis anormais das atividades reais e dos accruals dicionários}

A partir dos coeficientes apresentados nas Tabelas 1 e 2, são estimados os níveis anormais das atividades reais e dos accruals discricionários, ou seja, as proxies para gerenciamento de resultados contábeis.

Para calcular o total do gerenciamento de resultados por atividades reais (GRAR) foi utilizado o modelo proposto por Gunny (2010). Com os coeficientes encontrados pelo modelo Paulo (2007), para calcular o gerenciamento de resultados por escolhas contábeis, os accruals totais foram decompostos em discricionários e não discricionários, de acordo com o proposto por McNichols e Wilson (1988).

Ressalta-se que, caso o nível anormal de atividades reais ou dos accruals dicionários seja menor que zero $(\mathrm{GRAR}<0$ e GRAD $<0)$ indica que as empresas estão gerenciando seus resultados, por atividades reais e por accruals dicionários, com o intuito de aumentar o resultado contábil. As estatísticas descritivas das proxies de gerenciamento de resultados são apresentadas na Tabela 3. 
Jislene Trindade Medeiros, Edilson Paulo, Clayton Levy Lima de Melo, Renato Henrique Gurgel Mota

Tabela 3 - Estatística descritiva dos níveis anormais de atividades reais e dos accruals discricionários

\begin{tabular}{ccccccc}
\hline Variáveis & $\mathbf{N}^{\circ}$ Obs. & Mínimo & $\mathbf{1}^{\circ}$ Quartil & Mediana & $\mathbf{3}^{\circ}$ Quartil & Máximos \\
\hline AFCO & 1587 & $-2,449$ & $-0,058$ & $-0,004$ & 0,0538 & 5,495 \\
ADD & 1588 & $-1,037$ & $-0,077$ & $-0,038$ & 0,004 & 4,437 \\
APROD & 1500 & $-0,917$ & $-0,051$ & 0,019 & 0,072 & 0,999 \\
GRAR & 1486 & $-1,88$ & $-0,172$ & $-0,052$ & 0,103 & 3,734 \\
GRAD & 1343 & $-1,174$ & $-0,066$ & 0,022 & 0,023 & 2,007 \\
\hline
\end{tabular}

Fonte: Resultados da Pesquisa (2017).

Conforme os dados da mediana, apresentados na Tabela 3 , há indícios de que pelo menos $50 \%$ das empresas listadas na B3, no período de 2010 a 2015, parecem estar gerenciando os seus resultados por meio do fluxo de caixa operacional anormal (AFCO), utilizando descontos nos preços de venda ou condições de crédito mais facilidade, buscando aumentar o resultado contábil do período e, por meio das despesas discricionárias anormais (ADD), divulgando menos despesas discricionárias com o mesmo objetivo. Em relação aos níveis de produção anormais (APROD), infere-se que aproximadamente $25 \%$ das empresas listadas na B3, no período de 2010 a 2015, parecem estar interessadas em diminuir o resultado contábil, utilizando os níveis de produção.

No que se refere à variável "GRAR", referente à medida agregada dos níveis anormais de atividades reais, os resultados fornecem evidencias de que no mínimo metade das empresas investigadas, no período de 2010 a 2015, estão interessadas em aumentar o resultado contábil, resultado este convergente ao encontrado por Reis, Lamounier e Bressan (2015).

Analisando os dados da variável GRAD, que representa o total do gerenciamento por accruals discricionários, evidencia que, pelo menos $25 \%$ da amostra utilizam escolhas contábeis para aumentar o lucro do período, entretanto a partir do segundo quartil, ou seja, da mediana, as empresas utilizam escolhas contábeis com o objetivo de diminuir o lucro. Dessa forma, de modo divergente ao encontrado por Cupertino et al. (2015), verifica-se que essa estratégia de gerenciamento de resultados é utilizada por grande parte das empresas da amostra para diminuir o resultado contábil.

\subsection{Incentivo ao gerenciamento de resultados}

A análise da relação entre as estratégias de gerenciamento de resultados e as empresas que supostamente gerenciam resultados contábeis para evitar surpresa nos lucros foi realizada por meio

Tabela 4 - Incentivo ao gerenciamento de resultados

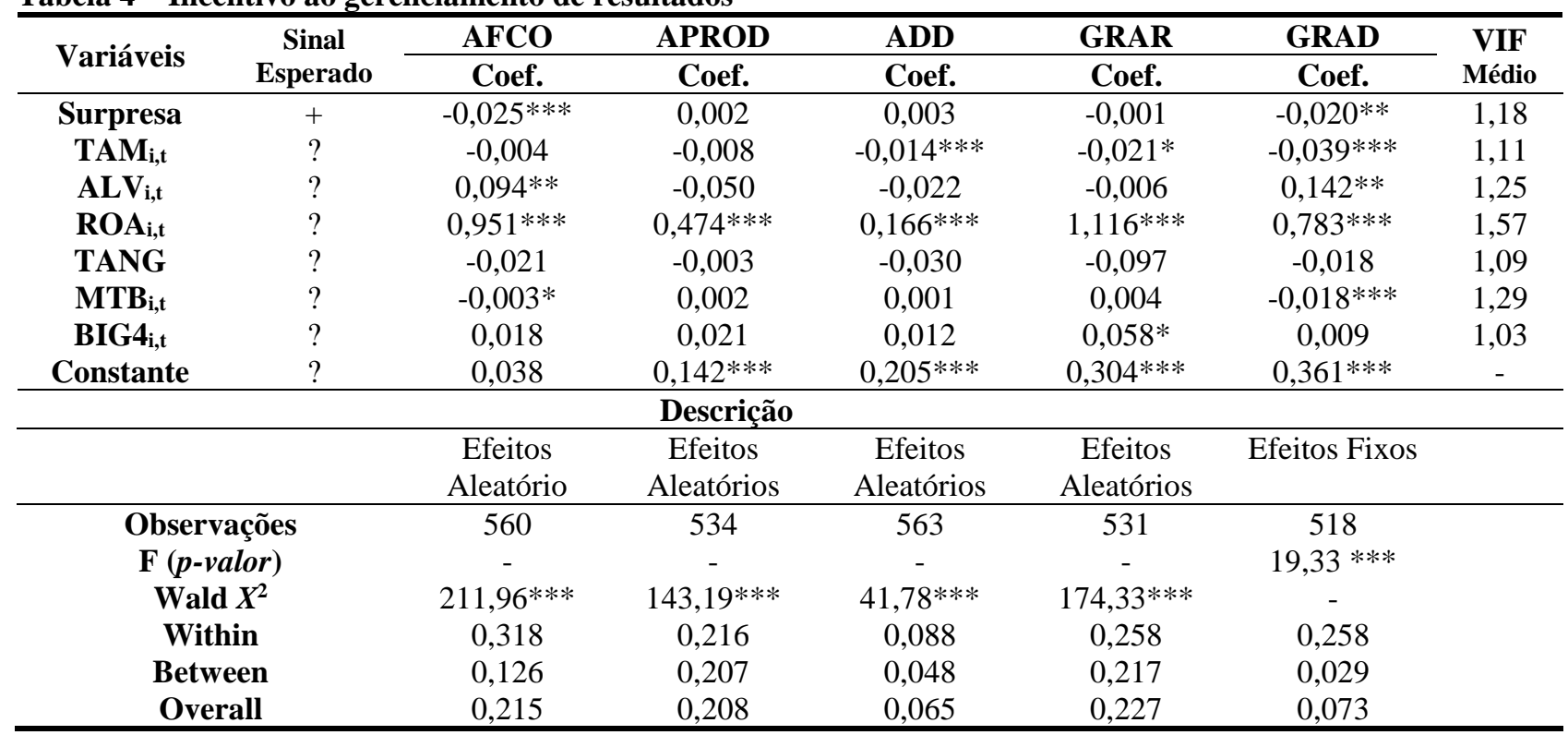

*,** e *** denotam, respectivamente, significância de $10 \%, 5 \%$ e $1 \%$.

Fonte: Resultados da Pesquisa (2017). 
de regressões pelo método pooling of independente cross section. Para tanto, as empresas que apresentaram erro de previsão de até $5 \%$ (Surpresa $\leq 5 \%$ ) foram consideradas suspeitas de gerenciar seus resultados, sendo identificadas por meio de uma variável dummy.

O coeficiente $\beta$ é utilizado para extrair evidências sobre o gerenciamento de resultados diante da hipótese testada e, conforme McNichols e Wilson (1988) se o $\beta$ estimado é significativamente maior que zero, pode-se dizer que existem evidências de que as empresas gerenciam os resultados contábeis por atividades reais e por accruals discricionários relacionadas a hipótese investigada.

Dessa forma, como o teste $\mathrm{F}$ e os testes de Wald $X^{2}$ apresentaram resultados significante a $1 \%$, logo, os coeficientes estimados são estatisticamente diferentes de zero. Observando o $p$-valor estimado para a variável "Surpresa" dos níveis anormais do Fluxo de Caixa Operacional (AFCO), verifica-se que este se mostrou significante ao nível de $1 \%$, contudo com sinal negativo. Portanto, quanto maior é o erro de previsão dos analistas financeiros menor o nível anormal do fluxo de caixa, resultado que contraria a hipótese de que essa estratégia de gerenciamento de resultados é utilizada para evitar surpresa nos lucros.

Verifica-se que os níveis anormais do volume de produção e das despesas discricionárias, assim com o total do gerenciamento de resultados por atividades reais não apresentaram coeficientes significantes. Assim, não há indícios de que as empresas que apresentam erros de previsão próximos ao lucro previsto pelos analistas, gerenciam seus resultados por atividades reais.

Conforme a Tabela 4, verifica-se que o coeficiente da variável "Surpresa" da regressão do nível anormal de accruals discricionários (GRAD) encontrado foi significante a 5\%, contudo não foi maior do que zero. Assim, não há indicativos de que os resultados são gerenciados por accruals discricionários para atingir o lucro previsto pelos analistas financeiros.

\subsection{Análise da utilização das estratégias de gerenciamento de resultados para evitar surpresa no lucro}

$\mathrm{Na}$ Tabela 5, são apresentadas algumas estatísticas descritivas referentes às variáveis operacionais utilizadas para responder ao problema de pesquisa, a fim de permitir uma compreensão geral da natureza da amostra.

Tabela 5 - Estatística descritiva das variáveis do teste da hipótese

\begin{tabular}{ccccccc}
\hline Variáveis & $\mathbf{N}^{\circ}$ Obs. & Média & $\begin{array}{c}\text { Desvio- } \\
\text { padrão }\end{array}$ & Mínimo & Mediana & Máximo \\
\hline SURPRESA & 667 & 0,021 & 1,332 & $-11,8$ & 0,013 & 14,1 \\
GRAR & 1.486 & $-0,008$ & 0,339 & $-1,877$ & $-0,052$ & 3,734 \\
GRAD & 1.343 & $-0,022$ & 0,137 & $-1,174$ & 0,022 & 2,007 \\
TAM & 1.617 & 7,392 & 1,952 & 0,612 & 7,52 & 13,710 \\
ALV & 1.601 & 0,313 & 0,306 & 0,000 & 0,289 & 4,339 \\
ROA & 1.508 & 0,025 & 0,441 & $-8,021$ & 0,029 & 2,18 \\
TANG & 1.579 & 0,279 & 0,240 & 0,000 & 0,234 & 0,991 \\
MTB & 1.190 & 2,350 & 3,89 & 0,0149 & 1,350 & 57,1 \\
BIG4 & 1.437 & - & - & - & - & - \\
\hline
\end{tabular}

Fonte: Resultados da Pesquisa (2017).

Entre os pontos de destaque, observa-se que a variável dependente "Surpresa" possui grande amplitude e, como pode ser verificado pela mediana, pelo menos $50 \%$ da amostra apresenta erros de previsão positivos, o que sinaliza o viés otimista dos analistas financeiros que atuam no mercado brasileiro, evidência essa, também documentada Martinez (2007).

Verifica-se na Tabela 6, que a variável referente ao gerenciamento de resultados por accruals (GRAD) possui significância estatística ao nível de $10 \%$ e com sinal positivo, sugerindo que o erro de previsão de lucro dos analistas financeiros é diretamente relacionado com os accruals discricionários, o que significa que quanto maior o nível dos accruals das empresas, maior é o erro 
médio da previsão. Esses resultados estão alinhados ao encontrado por Martins, Paulo e Monte (2016) no mercado brasileiro e, com o estudo de Ayers, Jiang e Yeung (2006), no que tange a influência dos accruals discricionários nas previsões dos analistas.

Tabela 6 - Regressão entre a surpresa nos lucros e as estratégias de gerenciamento de resultados

\begin{tabular}{|c|c|c|c|c|c|}
\hline Variáveis & $\begin{array}{c}\text { Sinal } \\
\text { Esperado }\end{array}$ & $\begin{array}{c}\text { GRAR } \\
\text { Coeficientes }\end{array}$ & $\begin{array}{c}\text { GRAD } \\
\text { Coeficientes }\end{array}$ & $\begin{array}{c}\text { GRAR e GRAD } \\
\text { Coeficientes }\end{array}$ & VIF \\
\hline GRAR $_{\mathrm{i}, \mathrm{t}}$ & - & $-0,078$ & - & $-0,008$ & 1,42 \\
\hline GRAD $_{i, t}$ & - & - & $0,899 * *$ & $0,893^{*}$ & 1,51 \\
\hline $\mathbf{T A M}_{\mathbf{i}, \mathbf{t}}$ & $?$ & $-0,049$ & $-0,045$ & $-0,046$ & 1,16 \\
\hline $\mathbf{A L V} \mathbf{V}_{\mathrm{i}, \mathrm{t}}$ & $?$ & $-0,459$ & $-0,358$ & $-0,362$ & 1,32 \\
\hline $\mathbf{R O A}_{i, t}$ & $?$ & $-4,231 * * *$ & $-4,745 * * *$ & $-4,738 * * *$ & 2,00 \\
\hline TANG & $?$ & $-0,302$ & $-0,173$ & $-0,173$ & 1,12 \\
\hline MTB $_{\mathrm{i}, \mathrm{t}}$ & $?$ & 0,002 & 0,002 & 0,002 & 1,49 \\
\hline BIG4 $4_{i, t}$ & $?$ & $-0,574 * * *$ & $-0,588 * * *$ & $-0,588 * * *$ & 1,04 \\
\hline Constante & $?$ & 2,033 & $1,969 * * *$ & $1,971 * * *$ & - \\
\hline \multicolumn{6}{|c|}{ Descrição } \\
\hline \multicolumn{2}{|c|}{ Observações } & 531 & 518 & 518 & \\
\hline \multicolumn{2}{|c|}{ F (p-valor) } & $6,26 * * *$ & $5,86 * * *$ & $5,40 * * *$ & \\
\hline \multicolumn{2}{|c|}{$\mathbf{R}^{2}$} & 0,1266 & 0,1222 & 0,1222 & \\
\hline \multicolumn{2}{|c|}{$\mathbf{R}^{2}$ Ajustado } & 0,1064 & 0,1013 & 0,0995 & \\
\hline
\end{tabular}

$*, * * \mathrm{e}^{* * *}$ denotam, respectivamente, significância de $10 \%, 5 \%$ e $1 \%$.

Regressões pelo método pooling of independente cross section com dummies para ano.

Fonte: Resultados da Pesquisa (2017).

Observa-se que a variável correspondente ao gerenciamento de resultados por atividades reais não apresentou significância estatística. Assim, os resultados encontrados são divergentes aos encontrados por Roychowdhury (2006) e, dessa forma, não se pode dizer que as empresas manipulam suas atividades reais para evitar reportar surpresa em relação às previsões dos analistas.

Conforme a Tabela 6, a variável de controle rentabilidade sobre o ativo (ROA) e a variável dummy que indica a empresa ser ou não auditada por uma das 4 maiores empresas de auditoria do mundo (BIG4) foram significantes a $1 \%$ e com sinal negativo. Assim, há indícios de que quanto maior a rentabilidade das empresas menor é o erro de previsão dos analistas e que os analistas preveem lucros mais próximos do real quando a empresas é auditada por uma das big four.

$\mathrm{Na}$ Tabela 6 também são apresentados os resultados de regressões que analisam individualmente a influência de cada uma das estratégias de gerenciamento de resultados na surpresa dos lucros. Como pode ser visualizado, os resultados encontrados são convergentes ao encontrado quando analisada a influência conjunta do gerenciamento de resultados por atividades reais e por accruals discricionários na surpresa dos lucros.

Portanto, não há indícios de que as empresas utilizaram o gerenciamento de resultados por accruals discricionários e por atividades reais para evitar surpresa nos lucros em relação ao lucro previsto pelos analistas financeiros no período de 2010 a 2015.

\section{CONSIDERAÇÕES FINAIS}

Esta pesquisa objetivou verificar se as empresas com ações negociadas no mercado acionário brasileiro, no período de 2010 a 2015, utilizaram no mesmo exercício físcal o gerenciamento de resultados por atividades reais e por accruals discricionários, a fim de evitar divulgar surpresa nos lucros em relação as metas de resultados previstas pelos analistas financeiros.

Ao estimar o gerenciamento de resultados total por meio das atividades reais, foi observado que mais de $50 \%$ das empresas manipulam os números contábeis por meio dessa estratégia de gerenciamento, com o objetivo de aumentar o resultado contábil do período corrente, indicando que as empresas podem estar preocupadas em atingir um lucro alvo no período e, consequentemente, evitar apresentar perdas associadas ao não atendimento de uma meta de lucros predefinida. 
Os resultados revelaram que as empresas que apresentam erros de previsão muito próximos ao lucro previsto pelos analistas financeiros não possuem incentivos para gerenciar seus resultados por atividades reais e por accruals discricionários. Assim, não há indícios de que as empresas da amostra gerenciaram seus resultados para atingir o lucro previsto pelos analistas e evitar divulgar surpresa nos lucros.

Em se tratando da utilização das estratégias de gerenciamento de resultados para evitar divulgar surpresa em relação a meta de lucros prevista pelos analistas financeiros, foi verificado que o nível dos accruals discricionários das empresas da amostra relacionam-se positivamente com o erro da previsão, sugerindo que as empresas listadas na B3, no período de 2010 a 2015, não utilizaram essa estratégia de gerenciamento de resultados para diminuir a surpresa nos lucros em relação a previsão de lucros proferidas pelos analistas financeiros. Como não foi evidenciado um relacionamento significante entre o nível de gerenciamento de resultados por atividades reais e a surpresa nos lucros, é possível afirmar que não são adotadas estratégias de manipulação de atividades reais para que as previsões de lucros dos analisas sejam atendidas. Dessa forma, apesar de ter sido observado que mais de $50 \%$ das empresas analisadas gerenciam seus resultados, por atividades reais com a finalidade de aumentar seus resultados contábeis, tal fato não contribui para o atendimento as previsões de lucros realizadas pelos analistas financeiros.

Portanto, rejeita-se a hipótese norteadora da pesquisa de que $\left(\mathrm{H}_{1}\right)$ as empresas gerenciam seus resultados por atividades reais e por accruals discricionários para evitar surpresa nos lucros, já que, de acordo com os resultados, não há indícios de que são adotadas no mesmo exercício fiscal as duas estratégias de gerenciamento de resultados contábeis, para atingir as previsões dos lucros dos analistas e, consequentemente, para evitar divulgar surpresa nos lucros em relação a esse benchmarking.

Este estudo contribui para subsidiar discussões sobre a utilização conjunta de estratégias de gerenciamento de resultados contábeis para alcançar uma meta de lucro desejada, especificamente para o atingir o resultado previsto pelos analistas financeiros e atender as expectativas do mercado. Além disso, fornece evidências de que possivelmente os gestores das empresas do mercado de capitais brasileiro não se preocupam em evitar surpresa nos lucros, a ponto de gerenciarem seus resultados por atividades reais e por accruals discricionários com essa finalidade e que, esses intermediários de informação não evitam que os gestores adotem práticas oportunistas de manipulação das informações contábeis. Outra constatação é que a rentabilidade e a qualidade da auditoria possuem uma relação inversa com a surpresa nos lucros.

\section{REFERÊNCIAS}

Almadi, M., \& Lazic, P. (2016). CEO incentive compensation and earnings management: The implications of institutions and governance systems. Management Decision, 54(10), 2447-2461.

Ardison, K. M. M., Martinez, A. L., \& Galdi, F. C. (2012). The effect of leverage on earnings management in Brazil. Advances in Scientific and Applied Accounting, 5(3), 305-324.

Ayers, B. C., Jiang, J., \& Yeung, P. E. (2006). Discretionary accruals and earnings management: An analysis of pseudo earnings targets. The Accounting Review, 81(3), 617-652.

Ayers, B. C., Jiang, J., \& Yeung, P. E. (2006). Discretionary accruals and earnings management: An analysis of pseudo earnings targets. The Accounting Review, 81(3), 617-652.

Barth, M. E., Elliott, J. A., \& Finn, M. W. (1999). Market rewards associated with patterns of increasing earnings. Journal of Accounting Research, 37(2), 387-413.

Bartov, E., Givoly, D., \& Hayn, C. (2002). The rewards to meeting or beating earnings expectations. Journal of Accounting and Economics, 33(2), 173-204.

Becker, C. L., DeFond, M. L., Jiambalvo, J., \& Subramanyam, K. R. (1998). The effect of audit quality on earnings management. Contemporary Accounting Research, 15(1), 1-24.

Bekiris, F. V., \& Doukakis, L. C. (2011). Corporate governance and accruals earnings management. Managerial and Decision Economics, 32(7), 439-456.

Bowen, R. M., Rajgopal, S., \& Venkatachalam, M. (2008). Accounting discretion, corporate governance, and firm performance. Contemporary Accounting Research, 25(2), 351-405. 
Brown, L. D., \& Caylor, M. L. (2005). A temporal analysis of quarterly earnings thresholds: Propensities and valuation consequences. The Accounting Review, 80(2), 423-440.

Call, A. C., Chen, S., Miao, B., \& Tong, Y. H. (2014). Short-term earnings guidance and accrual-based earnings management. Review of Accounting Studies, 19(2), 955-987.

Cupertino, C. M., Martinez, A. L., \& da Costa Jr, N. C. (2015). Earnings manipulations by real activities management and investors' perceptions. Research in International Business and Finance, 34, 309323.

Dechow, P., Ge, W., \& Schrand, C. (2010). Understanding earnings quality: A review of the proxies, their determinants and their consequences. Journal of Accounting and Economics, 50(2-3), 344-401.

Degeorge, F., Patel, J., \& Zeckhauser, R. (1999). Earnings management to exceed thresholds. The Journal of Business, 72(1), 1-33.

Dutra, E. S., \& da Costa, F. M. (2014). A relação entre gerenciamento de resultados e conservadorismo contábil em companhias abertas brasileiras. Advances in Scientific and Applied Accounting, 7(1), 149-170.

Grecco, M. C. P. (2013). The Effect of Brazilian convergence to IFRS on earnings managment by listed Brazilian nonfinancial companies. Brazilian Business Review, 10(4), 110-132.

Gunny, K. A. (2010). The relation between earnings management using real activities manipulation and future performance: Evidence from meeting earnings benchmarks. Contemporary Accounting Research, 27(3), 855-888.

Healy, P. M., \& Wahlen, J. M. (1999). A review of the earnings management literature and its implications for standard setting. Accounting Horizons, 13(4), 365-383.

Kasznik, R., \& McNichols, M. F. (2002). Does meeting earnings expectations matter? Evidence from analyst forecast revisions and share prices. Journal of Accounting Research, 40(3), 727-759.

Koh, P. S. (2003). On the association between institutional ownership and aggressive corporate earnings management in Australia. The British Accounting Review, 35(2), 105-128.

Macedo, M. A. S., \& Kelly, V. L. A. (2016). Gerenciamento de resultados em instituições financeiras no Brasil: uma análise com base em provisões para crédito de liquidação duvidosa. Revista Evidenciação Contábil \& Finanças, 4(2), 82-96.

Martinez, A. L. (2006). Como o mercado de capitais brasileiro reage a surpresa nos lucros?. REAd-Revista Eletrônica de Administração, 12(3), 1-23.

Martinez, A. L. (2013). Gerenciamento de resultados no Brasil: um survey da literatura. BBR-Brazilian Business Review, 10(4), 1-31.

Martinez, A. L. (2007). Otimismo e viés de seleção dos analistas. BBR-Brazilian Business Review, 4(2), 104-118.

Martinez, A. L., \& Cardoso, R. L. (2009). Gerenciamento da informação contábil no Brasil mediante decisões operacionais. REAd-Revista Eletrônica de Administração, 15(3), 600-626.

Martinez, A. L. (2011). The role of analysts as gatekeepers: enhancing transparency and curbing earnings management in Brazil. Revista de Administração Contemporânea, 15(4), 712-730.

Martins, V. G., Paulo, E., \& do Monte, P. A. (2016). O gerenciamento de resultados contábeis exerce influência na acurácia da previsão de analistas no Brasil?. Revista Universo Contábil, 12(3), 73-90.

McNichols, M., \& Wilson, G. P. (1988). Evidence of earnings management from the provision for bad debts. Journal of Accounting Research, 1-31.

Mota, R. H. G., da Cunha, A. C., de Oliveira, A. F., \& Paulo, E. (2017). Previsão de lucro e gerenciamento de resultados: evidências empíricas no mercado acionário brasileiro. Revista Universo Contábil, 13(1), 6-26.

Myers, J. N., Myers, L. A., \& Skinner, D. J. (2007). Earnings momentum and earnings management. Journal of Accounting, Auditing \& Finance, 22(2), 249-284.

Paulo, E. (2007). Manipulação das informações contábeis: uma análise teórica e empírica sobre os modelos operacionais de detecção de gerenciamento de resultados (Tese de Doutorado). Universidade de São Paulo, Programa de Pós-Graduação em Controladoria e Contabilidade, São Paulo.

Paulo, E., \& Mota, R. H. G. (2019). Business cycles and earnings management strategies: a study in Brazilian public firms. Revista Contabilidade \& Finanças, (AHEAD). 
Persakis, A., \& Iatridis, G. E. (2015). Cost of capital, audit and earnings quality under financial crisis: A global empirical investigation. Journal of International Financial Markets, Institutions and Money, 38, 3-24.

Reis, E. M., da Cunha, J. V. A., \& Ribeiro, D. M. (2014). Análise do gerenciamento de resultados por meio de decisões operacionais nas empresas componentes do IBrX-Índice Brasil. Advances in Scientific and Applied Accounting, 7(2), 201-223.

Reis, E. M., Lamounier, W. M., \& Bressan, V. G. F. (2015). Evitar Divulgar Perdas: Um Estudo Empírico do Gerenciamento de Resultados por Meio de Decisões Operacionais. Revista Contabilidade \& Finanças-USP, 26(69), 247-260.

Roychowdhury, S. (2006). Earnings management through real activities manipulation. Journal of Accounting and Economics, 42(3), 335-370.

Schipper, K., \& Vincent, L. (2003). Earnings quality. Accounting horizons, 17, 97-110.

Almeida-Santos, P. S., Verhagem, J. A., \& Bezerra, F. A. (2011). Gerenciamento de resultados por meio de decisões operacionais e a governança corporativa: análise das indústrias siderúrgicas e metalúrgicas brasileiras. Revista de Contabilidade e Organizações, 5(13), 55-74.

Watts, R. L., \& Zimmerman, J. L. (1990). Positive accounting theory: a ten year perspective. The Accounting Review, 131-156.

Yu, F. F. (2008). Analyst coverage and earnings management. Journal of Financial Economics, 88(2), 245271.

Zang, A. Y. (2011). Evidence on the trade-off between real activities manipulation and accrual-based earnings management. The Accounting Review, 87(2), 675-703. 